\title{
Efecto del injerto y del bioestimulante Fartum® sobre la producción y calidad en tomate cherry
}

\author{
Effect of grafting and the biostimulant Fartum ${ }^{\circledR}$ on production and \\ quality in cherry tomatoes
}

\author{
Pilar Mazuela A. ${ }^{1}$, Bárbara Cepeda, Victoria Cubillos
}

\begin{abstract}
RESUMEN
La producción de hortalizas en la Región de Arica y Parinacota ha adoptado nuevas tecnologías en protección de cultivos, riego y variedades híbridas para aumentar el rendimiento y mejorar la calidad de sus productos, considerando las características de suelo y clima que se observan en los valles costeros de la Región. Según el último censo agropecuario, el tomate es la hortaliza de mayor importancia económica regional, tanto por superficie cultivada como por rendimiento y por época de producción. Actualmente se están haciendo diversos estudios en el uso de bioestimulantes para mejorar la nutrición de las plantas y la tolerancia al estrés hídrico. El objetivo de este trabajo fue evaluar el efecto de bioestimulante natural a base a algas marinas (Fartum®) sobre los parámetros de fertirriego, producción y calidad de un cultivo de tomate cherry var. Bambino. Se realizaron dos ensayos, uno con plantas francas y otro con plantas injertadas (portainjerto Multifort). Los resultados sugieren que el uso de Fartum ${ }^{\circledR}$ mejora el rendimiento de tomate cherry en plantas francas y no se observan diferencias significativas al aplicar bioestimulante en plantas injertadas. Se concluye que las plantas francas con aplicación de bioestimulante logran un rendimiento significativamente mayor, similar a las medias obtenidas con plantas injertadas, lo que sugiere que el uso de productos naturales en base a algas marinas tiene un efecto similar al injerto.
\end{abstract}

Palabras claves: cultivo sin suelo, Solanum lycopersicum L., bioestimulante, injerto.

\section{SUMMARY}

Vegetable production in the Region de Arica y Parinacota, Chile, has adopted new technologies in crop production, irrigation and hybrid varieties, to increase yield and improve the quality of its products, taking into account the soil and climate characteristics of the coastal valleys of this region. According to the last agricultural census, tomatoes are the most important vegetable in the regional economy, both in area cultivated and in yield and production period. Currently there are a number of studies underway on the use of biostimulants to improve plant nutrition, salt tolerance water stress. The objective of this study was to evaluate the effect of a natural biostimulant based on marine algae (Fartum $\left.{ }^{\circledR}\right)$ on parameters offertigation, production and quality of the cherry tomato var. Bambino. Two trials were performed, one with entire plants and the other with grafted plants (Multifort rootstock). The results suggest that using Fartum $®$ improves the yield of cherry tomato in entire plants, although there was no significant difference of its application on grafted plants. We conclude that there was a significant increase in yield when this biostimulant was applied to entire plants, similar to the yield of grafted plants, which suggests that the use of natural products based on marine algae has an effect on this cultivar similar to that of grafting under conditions of salinity.

Key words: soilless culture, Solanum lycopersicum L., bioestimulant, graft.

\section{Introducción}

Uno de los ejes de desarrollo de la XV Región de Arica y Parinacota es la actividad agrícola, que se efectúa principalmente en los valles costeros de Azapa y Lluta. Estos valles presentan condiciones climáticas excepcionales para el cultivo de hortalizas durante todo el año, siendo el principal proveedor de hortalizas de la zona central durante el invierno (Saavedra y Tapia, 2009). Predominan las condiciones de clima de desierto costero con nubosidad abundante, ausencia de heladas, vientos moderados, alta humedad relativa y alta radiación solar directa, durante todo el año. La temperatura media anual es de $18{ }^{\circ} \mathrm{C}$, máximas medias anuales de $23,6^{\circ} \mathrm{C}$ y mínimas de $13,8^{\circ} \mathrm{C}$ (Torres y Acevedo, 2008). Sin embargo, dada su ubicación, distante a más de $2.000 \mathrm{~km}$ de los grandes centros urbanos

1 Universidad de Tarapacá, Chile. E-mail: pmazuela@uta.cl 
de Chile, la producción se concentra en hortalizas de alto valor, como el tomate, pimiento y poroto verde. La hortaliza de mayor importancia económica en la región es el tomate para consumo fresco que supera las 840 ha (INE, 2008). Los rendimientos superan significativamente las medias nacionales, alcanzando medias de $113 \mathrm{tha}^{-1}$ en tomate, superior a la media nacional de $71 \mathrm{t} \mathrm{ha}^{-1}$ (INE, 2010). Los productores de tomate han ido adoptando nuevas tecnologías para aumentar el rendimiento y mejorar el proceso de producción. Entre las mejoras que se han incorporado, el uso de la técnica del injerto para mejorar la tolerancia de las plantas a los nematodos y las enfermedades del suelo incrementa la resistencia a la sequía y mejora la absorción de agua y nutrientes, cuyo resultado final es un mayor vigor en la planta, favoreciendo con ello el desarrollo de la agricultura sustentable del futuro (López-Elías et al., 2008). Otra alternativa es la sustitución de agroquímicos tóxicos o de difícil degradación por insumos más amigables con el medio ambiente conocidos como biofertilizantes y/o bioestimulantes. Estos productos contienen aminoácidos, ácidos húmicos y/o fitohormonas de fácil disponibilidad, cuya absorción no depende de la fotosíntesis y disminuye el consumo energético de la planta (Parrado et al. (2008). Algunos efectos del uso de bioestimulantes son descritos por Vasconcelos et al. (2009), como el aumento de la retención de agua de la hoja y el metabolismo antioxidante cuando la planta es sometida a estrés hídrico. La demanda por insumos agrícolas que no contaminen el medio ambiente ha estimulado el uso de productos a base de algas marinas como bioestimulante de raíces. Sin embargo, se corre el riesgo de que, debido a su fácil accesibilidad, un aumento en su valor económico genere una sobreexplotación de las algas marinas (Ugarte y Sharp, 2012).

El objetivo de esta investigación es evaluar el efecto de la aplicación de un bioestimulante natural en base a algas marinas, sobre los parámetros de fertirriego, producción y calidad en un cultivo de tomate tipo cherry en plantas francas e injertadas.

\section{Materiales y Métodos}

El ensayo del cultivo de tomate tipo cherry se realizó en la Facultad de Ciencias Agronómicas de la Universidad de Tarapacá ubicada en el kilómetro 12 del Valle de Azapa, a 250 m.s.n.m., en la XV Región de Arica y Parinacota. El cultivo se estableció al interior de un invernadero de malla antiáfido, con una superficie de $200 \mathrm{~m}^{2}$ en cultivo sin suelo, utilizando compost de residuos agroindustriales como sustrato. El sustrato fue acondicionado según describe Mazuela et al. (2005) y Mazuela y Urrestarazu (2009).

Se realizaron dos ensayos, uno con plantas francas de tomate tipo cherry variedad Bambino $\mathrm{y}$ otro con plantas injertadas de tomate tipo cherry variedad Bambino sobre Multifort. En ambos casos los tratamientos fueron: T0, sin bioestimulante; T1, con aplicación de bioestimulante. Se utilizó un bioestimulante natural a base de algas marinas, Fartum ${ }^{\circledR}$. La aplicación de bioestimulante se realizó de forma manual, en dosis de $0,46 \mathrm{~mL}$ de Fartum ${ }^{\circledR}$ por cada planta. Esta dosis fue calculada según las recomendaciones del producto para el cultivo de tomate y se realizó diariamente durante los primeros cinco días después del trasplante, y luego cada 15 días.

El tomate tipo cherry tuvo un ciclo de inviernoprimavera, el trasplante se realizó el 30 de agosto de 2011. La densidad de plantación fue de 1,33 plantas por $\mathrm{m}^{-2}$ colocadas en contenedores de sustrato de $2 \mathrm{~L}$, con una planta por saco. Las plantas fueron conducidas a un eje y se usaron abejorros (Bombus terrestris) para la polinización. La conductividad eléctrica (CE) media del agua de riego fue de $0,8 \mathrm{dS} \mathrm{m}^{-1}$ y $\mathrm{pH}$ 8,3. La composición química del agua de riego (en $\mathrm{mmol} \mathrm{L}^{-1}$ ) fue: 0,1 de $\mathrm{NO}_{3}^{-} ; 2,9$ de $\mathrm{HCO}_{3}^{-} ; 2,3$ de $\mathrm{SO}_{4}^{2-} ; 3,3 \mathrm{de}^{-}$;

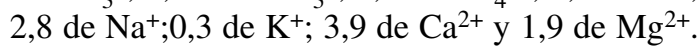
La CE de la disolución nutritiva utilizada fue de 2,3 dS m ${ }^{-1}$ y $\mathrm{pH} 7,2$. La composición media de los nutrientes aportados fue: 13 de $\mathrm{NO}_{3}^{-} ; 1,75 \mathrm{de}$ $\mathrm{H}_{2} \mathrm{PO}_{4}^{-} ; 2,5$ de $\mathrm{SO}_{4}{ }^{2-} ; 7,5$ de $\mathrm{K}^{+} ; 8$ de $\mathrm{Ca}^{2+}$ y 2,5 de $\mathrm{Mg}^{2+}$, expresados en $\mathrm{me} \mathrm{L}^{-1}$.

Para el análisis de producción y calidad se realizaron cosechas semanales comenzando el 11 de noviembre hasta el 30 de diciembre del 2011. Los parámetros de fertirriego analizados fueron: conductividad eléctrica $\left(\mathrm{dS} \mathrm{m}^{-1}\right), \mathrm{pH}$, porcentaje de drenaje y consumo hídrico $\left(\mathrm{L} \mathrm{m}^{-2}\right)$. Se evaluó la producción en $\mathrm{kg}$ y número de frutos $\mathrm{m}^{-2}$. La calidad de frutos se cuantificó peso $(\mathrm{g})$, diámetro $(\mathrm{mm})$ y firmeza $(\mathrm{kg})$ de fruto y sólidos solubles totales ( ${ }^{\circ}$ Brix) de frutos. La firmeza de fruto a la presión se determinó con un penetrómetro marca Effegi modelo FT 011 (0-11 lb), utilizando un émbolo de $8 \mathrm{~mm}$ de diámetro. La concentración de sólidos solubles se midió con un refractómetro portátil termocompensado marca Arquimed modelo 2003319. El diseño experimental 
para cada experimento fue de bloques completamente al azar (Little y Hills, 1976; Petersen, 1994) con dos tratamientos y cinco repeticiones. Para la separación de medias se usó la probabilidad asociada a la $t$ de Student. Para los cálculos se utilizó el programa Microsoft Excel 2000.

\section{Resultados y Discusión}

A continuación se presentan los resultados obtenidos durante el ciclo del cultivo de 120 días, de los dos ensayos realizados.

\section{Parámetros de fertirriego}

Los parámetros de fertirriego para el experimento con plantas francas e injertadas (Tabla 1) no muestra diferencia significativa entre tratamientos para conductividad eléctrica y $\mathrm{pH}$ del drenaje. Sin embargo, se observa una diferencia significativa en el menor porcentaje de drenaje y mayor consumo hídrico en plantas donde se aplicó bioestimulante, tanto en plantas francas como injertadas. Al comparar estos parámetros con los de Mazuela et al. (2012), se observan valores medios superiores en la conductividad eléctrica del drenaje y porcentaje de drenaje. Esto podría explicarse por la CE inicial del compost y un menor consumo hídrico medio durante el cultivo, lo que sugiere que las temperaturas medias fueron mas bajas. Estos resultados coinciden con los obtenidos por Caniguante et al. (2009) donde se observa una acción mitigadora en los efectos negativos de la salinidad al aplicar el bioestimulante.

\section{Parámetros de producción de tomate cherry}

Los parámetros de producción para ambos experimentos se observan en la Tabla 2. En el experimento con plantas francas se observa una mayor producción $\left(\mathrm{g} \mathrm{m}^{-2}\right)$ de tomates cherry al aplicar bioestimulante, esto coincide con los resultados obtenidos por Parrado et al. (2007) y Zodape et al. (2011) al evaluar el efecto de los bioestimulantes en la producción de tomate. No se observaron diferencias significativas en las plantas injertadas para la producción de tomates. Vasconcelos et al. (2009) sugieren que bajo condiciones de salinidad los bioestimulantes disminuyen el estrés hídrico, coincidiendo con los resultados de mayor consumo hídrico en plantas francas con bioestimulante (Tabla 1) y la mayor producción de frutos (Tabla 2).

Tabla 1. Variables de fertirriego para plantas francas (Experimento 1: var. Bambino) y plantas injertadas (Experimento 2: var. Bambino sobre Multifort) en cultivo de tomate cherry, según tratamiento: sin bioestimulante (T0) y con bioestimulante (T1).

\begin{tabular}{|c|c|c|c|c|c|c|c|c|}
\hline & \multicolumn{4}{|c|}{ Var. Bambino } & \multicolumn{4}{|c|}{ Var. Bambino sobre Multifort } \\
\hline & $\mathrm{CE}$ & $\mathrm{pH}$ & PD & $\mathrm{CH}$ & $\mathrm{CE}$ & $\mathrm{pH}$ & PD & $\mathrm{CH}$ \\
\hline T0 & 6,27 & 7,83 & 26,82 & 145 & 5,92 & 8,02 & 25,87 & 148 \\
\hline $\mathrm{T} 1$ & 6,31 & 7,88 & 23,66 & 152 & 6,00 & 7,84 & 23,05 & 154 \\
\hline$p$ & ns & ns & $*$ & $*$ & ns & ns & $*$ & $*$ \\
\hline
\end{tabular}

$*, * *, * * *, \mathrm{~ns}$, son $\mathrm{p} \leq 0,05, \mathrm{p} \leq 0,01, \mathrm{p} \leq 0,001$ y no significativo.

$\mathrm{CE}$ : conductividad eléctrica (dS m$\left.{ }^{-1}\right)$; PD: porcentaje de drenaje (\%); $\mathrm{CH}$ : consumo hídrico ciclo ( $\left.\mathrm{L} \mathrm{m}^{-2}\right)$.

Tabla 2. Variables de producción para plantas francas (Experimento 1: var. Bambino) y plantas injertadas (Experimento 2: var. Bambino sobre Multifort) en cultivo de tomate cherry, según tratamiento: sin bioestimulante (T0) y con bioestimulante (T1).

\begin{tabular}{lccccc}
\hline & \multicolumn{2}{c}{ Var. Bambino } & & \multicolumn{2}{c}{ Var. Bambino sobre Multifort } \\
\cline { 2 - 3 } & $\mathrm{g} \mathrm{m}^{-2}$ & Frutos m & & $\mathrm{g} \mathrm{m}^{-2}$ & \multicolumn{2}{c}{ Frutos m$^{-2}$} \\
\hline T0 & 2.200 & 216 & & 2.389 & 243 \\
T1 & 2.540 & 240 & & 2.446 & 245 \\
$p$ & $*$ & $\mathrm{~ns}$ & $\mathrm{~ns}$ & $\mathrm{~ns}$ \\
\hline
\end{tabular}

$*, * *, * * *, \mathrm{~ns}$, son $\mathrm{p} \leq 0,05, \mathrm{p} \leq 0,01, \mathrm{p} \leq 0,001$ y no significativo. 
Tabla 3. Variables de calidad para plantas francas (Experimento 1: var. Bambino) y plantas injertadas

(Experimento 2: var. Bambino sobre Multifort) en cultivo de tomate cherry, según tratamiento: sin bioestimulante (T0) y con bioestimulante (T1).

\begin{tabular}{|c|c|c|c|c|c|c|c|c|}
\hline & \multicolumn{4}{|c|}{ Var. Bambino } & \multicolumn{4}{|c|}{ Var. Bambino sobre Multifort } \\
\hline & $\mathrm{P}$ & $\mathrm{D}$ & FF & SS & $\mathrm{P}$ & $\mathrm{D}$ & $\mathrm{FF}$ & SS \\
\hline T0 & 10,2 & 25,4 & 2,06 & 9,08 & 9,8 & 27,0 & 2,04 & 9,56 \\
\hline $\mathrm{T} 1$ & 10,6 & 26,7 & 2,09 & 8,97 & 10,0 & 26,9 & 2,09 & 9,16 \\
\hline$p$ & $\mathrm{~ns}$ & $\mathrm{~ns}$ & $\mathrm{~ns}$ & $\mathrm{~ns}$ & $\mathrm{~ns}$ & $\mathrm{~ns}$ & ns & $*$ \\
\hline
\end{tabular}

$*, * *, * * *, \mathrm{~ns}$, son $\mathrm{p} \leq 0,05, \mathrm{p} \leq 0,01, \mathrm{p} \leq 0,001$ y no significativo.

P: peso (g fruto $\left.{ }^{-1}\right)$; D: diámetro $(\mathrm{mm})$; FF: firmeza de fruto $(\mathrm{Kg})$; SS: sólidos solubles $\left({ }^{\circ}\right.$ Brix $)$.

\section{Parámetros de calidad de frutos}

Los parámetros de calidad para ambos experimentos se observan en la Tabla 3. El peso medio de frutos es semejante a los obtenidos por Mazuela $e t$ al. (2010, 2012). No existe diferencia significativa en ninguno de los parámetros analizados para el experimento con plantas francas. Se observó que existe un mayor contenido de sólidos solubles en las plantas injertadas sin bioestimulante, debido al aumento de la salinidad en el medio radical (Dorais et al., 2001). Este fenómeno podría ser explicado por una disminución en la acumulación de agua en el fruto ya que al incrementarse la concentración de solutos en el agua de riego el potencial hídrico se reduce y las plantas experimentan dificultades para absorberla (Goykovic y Saavedra, 2007).

\section{Conclusión}

Los resultados del efecto de la aplicación de un bioestimulante natural en base a algas marinas tienen un efecto positivo en consumo hídrico y producción de frutos en plantas francas, alcanzando rendimientos semejantes a los rendimientos de plantas injertadas. En plantas injertadas también se ve un efecto positivo al consumo hídrico al aplicar un bioestimulante, sin embargo, no se observa una mayor producción. En el caso de la calidad de frutos, se observa un mayor contenido de sólidos solubles en plantas injertadas sin bioestimulante. Los resultados de este ensayo siguieren que la aplicación de bioestimulantes aumenta la eficiencia hídrica del cultivo de tomate y los rendimientos medios alcanzan valores semejantes al rendimiento de plantas injertadas.

\section{Agradecimientos}

Este trabajo ha sido financiado por el Proyecto de Investigación Estudiantil UTA 9723-11.

\section{Literatura Citada}

Caniguante, S.; Pizarro, L.; Pacheco, P. y Bastías, E. 2009. Respuesta de los cvs. de tomate (Solanum lycopersicum L.) "Poncho Negro" y Naomi en diferentes condiciones de crecimiento y la aplicación de un bioestimulante natural Fartum ${ }^{\circledR}$ en condiciones de salinidad. Idesia 27 (3): 19-28.

Dorais, M.; Papadopoulos, A.; Gosselin, A.

2001. Influence of electrical conductivity management on greenhouse tomato yield and fruit quality. Agronomie 21: 367-383.

Goykovic, V. y Saavedra, G.

2007. Algunos efectos de la salinidad en el cultivo del tomate y prácticas agronómicas de su manejo. Idesia 25 (3): 47-58.

Instituto Nacional de Estadísticas.

2008. VII Censo Agropecuario y Forestal 2006-2007. Resultados preliminares. INE Ediciones, Santiago de Chile, 444 p.
Instituto Nacional de Estadísticas

2010. Información Hortícola. Publicación Especial 20082009. INE Ediciones, Santiago de Chile, 128 p.

Little, T.; Hills, F.

1976. Métodos estadísticos para la investigación en la agricultura. Ed. Trillas, México, 270 p.

López-Elías, J.; Romo, A. y Domínguez, J.

2008. Evaluación de métodos de injerto en sandía (Citrullus lanatus (Thunb.) Matsum. \& Nakai) sobre diferentes patrones de calabaza. Idesia 26 (2): 13-18.

Mazuela, P.; Acuña, L.; Alvarez, M. y Fuentes, A.

2010. Producción y calidad de un tomate cherry en dos tipos de invernadero en cultivo sin suelo. Idesia 28 (2): 97-100.

Mazuela, P.; Salas, M.C. and Urrestarazu, M.

2005. Vegetable waste compost as substrate for melon. Commun. Soil Sci. Plant Anal. 36 (11-12): 1557-1572. 
Mazuela, P.; Trevizán, J. y Urrestarazu, M.

2012. A comparison of two types of agrosystems for the protected soilless cultivation of tomato crops in arid zones. J. Food Agric. Environ. 10 (1): 338-341.

Mazuela, P. y Urrestarazu, M.

2009. The effect of amendment of vegetable waste compost used as substrate in soilless culture on yield and quality of melon crops. Compost Sci. \& Utiliz., 17: 103-107.

Parrado, J.; Bautista, J.; Romero, E.J.; García-Martínez, A.M.; Friaza, V. y Tejada, M.

2008. Production of a carob enzymatic extract: Potential use as a biofertilizer. Bioresource Technology 99: 23122318.

Petersen, R.G. 1994. Agricultural Field Experiments. Marcel Dekker Inc., New York, 409 p.

Saavedra, A. y Tapia, L.

2009. Evaluación de las estrategias de producción de tomate (Lycopersicon esculentum Mill) fuera de estación para la agricultura de la provincia de Arica, Chile, entre los años 1995-2005. Idesia 27 (2): 91-96.

Torres, A. y Acevedo, E.

2008. El problema de salinidad en los recursos suelo y agua que afectan el riego en los valles de Lluta y Azapa en el norte de Chile. Idesia 26 (3): 31-44.

Ugarte, R. y Sharp, G. 2012. Management and production of the brown algae Ascophyllum nodosum in the Canadian maritimes. J Appl Phycol, 24: 409-416.

Vasconcelos, A.; Zhang, X.; Ervin, E. y Kiehl, J.

2009. Enzymatic antioxidant responses to bioestimulants in maize and soybean subjected to drought. Sci. Agric., 66 (3): 395-402.

Zodape, S.T.; Gupta, A.; Bhandari, S.C.; Rawat, U.S.; Chaudhary, D.R.; Eswaran, K. y Chikara, J.

2011. Foliar application of seaweed sap as bioestimulant for enhancement of yield and quality of tomato (Lycopersicon esculentum Mill.). J Sci Ind Res India, 70 (3): 215-219. 
